Orthopäde 2022 · 51:138-145

https://doi.org/10.1007/s00132-021-04148-y

Angenommen: 2. August 2021

Online publiziert: 9 . September 2021

(c) Der/die Autor(en) 2021

\section{Behandlung von Knochen- und Protheseninfektionen mit Bakteriophagen}

\author{
Ein systematisches Review
}

Zusammenfassung

Hintergrund: Die Behandlung von Knochen- und Protheseninfektionen bleibt trotz moderner Behandlungskonzepte mit interdisziplinärem Therapieansatz schwierig und weitere Maßnahmen zur Verbesserung des Behandlungsergebnisses sind wünschenswert. Präklinischen Studien liefern ein vielversprechendes Bild der Wirksamkeit von Bakteriophagen zur Behandlung von Knochen- und Protheseninfektionen.

Ziel der Arbeit: Die vorliegende Arbeit gibt eine systematische Übersicht über die klinische Anwendung von Bakteriophagen zur Behandlung von Knochen- und Protheseninfektionen.

Material und Methoden: Eine systematische Suche wurde in PubMed zur Identifikation von primären klinischen Daten zur Anwendung der Phagentherapie bei Patienten mit Knochen- und Protheseninfektion durchgeführt.

Ergebnisse: Elf Studien wurden eingeschlossen, bestehend aus 8 Fallberichten und 3 Fallserien. Indikationen der Phagentherapie waren periprothetische Infektionen ( $n=12,52,2 \%)$, frakturassoziierte Infektionen $(n=9,39,1 \%)$, Osteomyelitis $(n=1$, $4,4 \%$ ) und eine lliosakralgelenkinfektion nach Zementaugmentation einer Metastase $(n=1,4,4 \%)$. Die Interventionen waren heterogen, Phagen wurden intravenös verabreicht, intraoperativ ins Gelenk injiziert, intraoperativ lokal angewendet oder über Drainagen appliziert. In Kombination mit Antibiotikatherapie konnte eine vollständige Infekteradikation bei 18 Patienten (78,3\%) erreicht werden. Bei 91,3\% der Patienten wurden keine Nebenwirkungen berichtet.

Schlussfolgerung: Bakteriophagen sind eine vielversprechende Behandlungsmethode von Knochen- und Protheseninfektionen in Kombination mit einer Antibiotikatherapie. Zukünftige klinische Studien mit höherem Evidenzgrad werden benötigt, um eine erfolgreiche Translation der Bakteriophagentherapie in die klinische Praxis weiter zu etablieren.

\title{
Schlüsselwörter
}

Alternative Therapie · Antibiotikaresistenz $\cdot$ Phagentherapie $\cdot$ Protheseninfektion · BehandlungsOutcome

\section{Hinführung zum Thema}

Infektionen nach endoprothetischem Gelenkersatz oder Frakturosteosynthese sind eine Herausforderung in Orthopädie und Unfallchirurgie. Vor dem Hintergrund zunehmender Antibiotikaresistenzen rücken
Behandlungsalternativen zur etablierten Antibiotikatherapie in den Fokus. Insbesondere die Anwendung von Bakteriophagen scheint laut präklinischen Studien Wirksamkeit bei implantatassoziierten Infektionen zu besitzen. In diesem Beitrag wird eine Übersicht über primäre klinische 


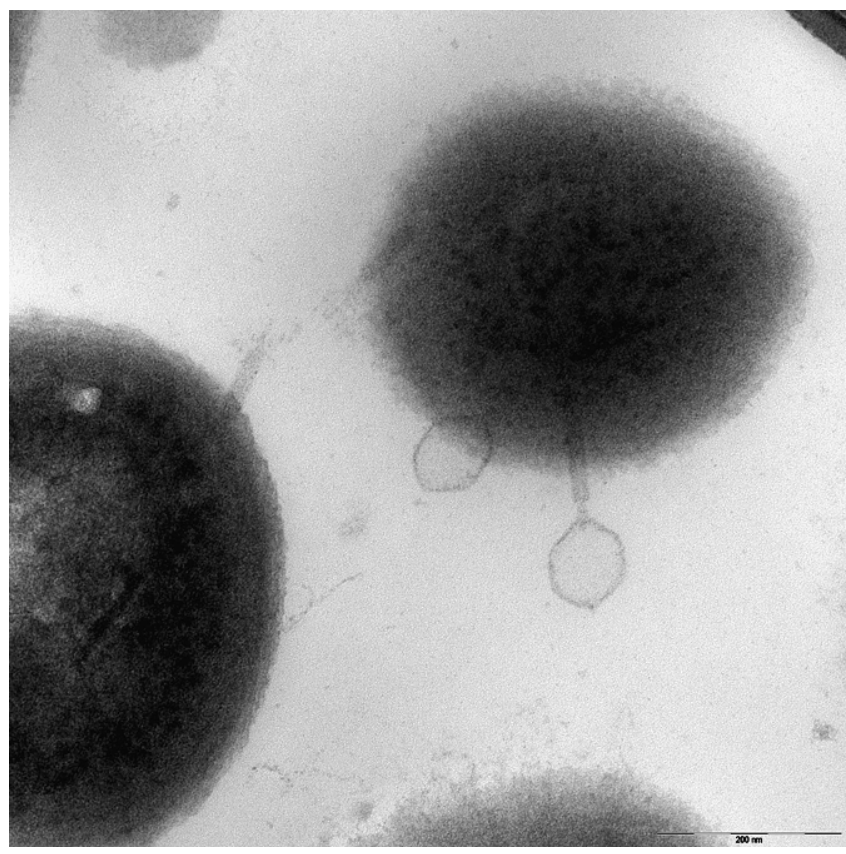

Abb. $1 \triangleleft$ Dargestellt sind Bakteriophagen an mit Staphylococcus aureus infizierten Osteoblasten. Aufgenommen mittels Transmissionselektronenmikroskopie (Skala $200 \mathrm{~nm}$ )

Daten zur Anwendung der Bakteriophagentherapie bei Patienten mit Knochenund Protheseninfektionen vorgestellt.

\section{Hintergrund und Fragestellung}

Der endoprothetische Gelenkersatz und die operative Frakturversorgung sind lebensverbessernde operative Verfahren für Millionen von Menschen auf der ganzen Welt. Neben den funktionellen Vorteilen, die eine Implantation von Endoprothesen oder Osteosynthesematerial bewirken können, besteht für jedes Implantat das Risiko einer Implantatinfektion. Ein Schlüsselelement in der Pathophysiologie von implantatassoziierten Knocheninfektionen ist die bakterielle Kolonisierung des Implantats und anschließende Biofilmbildung [29]. Schon frühe Studien identi-

\section{Abkürzungen}

\begin{tabular}{|c|c|}
\hline DTT & „Difficult-to-treat" \\
\hline FRI & $\begin{array}{l}\text { Frakturassoziierte Infektion } \\
\text { („fracture-related infection“) }\end{array}$ \\
\hline$M R S A$ & $\begin{array}{l}\text { Methicillin-resistenter Staphylococ- } \\
\text { cus aureus }\end{array}$ \\
\hline MSSA & $\begin{array}{l}\text { Methicillin-sensitiver Staphylococ- } \\
\text { cus aureus }\end{array}$ \\
\hline PFU & „Plaque forming units“ \\
\hline 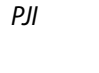 & $\begin{array}{l}\text { Periprothetische Gelenkinfektion } \\
\text { („periprosthetic joint infection“) }\end{array}$ \\
\hline PRISMA & $\begin{array}{l}\text { Prefered Reporting Items for } \\
\text { Systematic Reviews and Meta- } \\
\text { Analysis }\end{array}$ \\
\hline
\end{tabular}

fizierten das sogenannte „Race for the surface"-Phänomen, einen Wettstreit zwischen körpereigenen Zellen und Bakterien um die neue Besiedlungsoberfläche [14, 15]. Gerade die Infekteradikation nach Bildung eines chronischen Biofilms stellt eine besondere klinische Herausforderung dar. Die Diffusion von antimikrobiellen Wirkstoffen in Biofilme wird durch mehrere Faktoren wie die physikalische Barriere, den verstärkten Austausch von Antibiotikaresistenzgenen und langsamere Wachstumsraten deutlich eingeschränkt [1]. Infolgedessen sind Biofilmmikroorganismen bis zu 1000-mal resistenter gegen wachstumsabhängige antimikrobielle Wirkstoffe als ihr planktonisches Äquivalent [26, 30]. Entsprechend hängen Behandlungskonzepte für Knochen- und Protheseninfektionen hauptsächlich von der Dauer der Infektion ab, wobei der Reifungszustand des Biofilms als ein Hauptfaktor für die therapeutische Möglichkeit des Implantaterhaltes angesehen wird [36]. Gerade vor dem Hintergrund zunehmender Antibiotikaresistenzen wird nach Alternativen zur etablierten Antibiotikatherapie gesucht. Ein vielversprechender Ansatz sind Bakteriophagen, die ubiquitär in der Umwelt auftreten und zu den am häufigsten vorkommenden biologischen Organismen zählen (• Abb. 1; [5]). Diese Viren infizieren Bakterien selektiv, replizieren sich und werden schließlich durch
Lyse freigesetzt, wodurch der Wirt getötet wird.

Die Bakteriophagentherapie ist keine neue Behandlungsmethode. Felix d'Herelle hatte die Möglichkeit der zuvor vom Briten Twort im The Lancet beschriebenen Bakterienlyse für die klinische Anwendung erkannt und letztlich Shigellen-Enteritis bei französischen Truppen im 1. Weltkrieg erfolgreich behandelt. Er war es auch, der den Begriff „Bakteriophage" geprägt und aufgrund seiner Errungenschaften 1925 mit der Leeuwenhoek-Medaille ausgezeichnet wurde [4, 6]. Durch die Entdeckung des Penicillins war der Behandlungsansatz mit Bakteriophagen insbesondere in der westlichen Welt nach dem 2. Weltkrieg mehr oder weniger in Vergessenheit geraten. Indes wurden Bakteriophagen in der ehemaligen Sowjetunion zur Therapie verschiedener Infektionen, wie gastrointestinalen Infekten aber auch Gasbrand bei Soldaten, angewandt. Trotz der dortigen breiten Anwendung, sind nur wenige Publikationen in englischer Sprache verfügbar [27]. Ein Beispiel sind Veröffentlichungen des Instituts für Immunologie und Experimentelle Therapie, auch bekannt als "Hirszfeld Institut" im heutigen Polen, wo zwischen 1981 und 19991857 Fälle mit Bakteriophagen behandelt und 85-92,4\% erfolgreiche Ergebnisse berichtet wurden $[25,33]$.

Die zunehmende Ausbreitung der antimikrobiellen Resistenz, zusammen mit der Entwicklung von Analysetechniken wie Hochdurchsatzsequenzierungen und Elektronenmikroskopie, die es ermöglichen Phagen genauer zu untersuchen, führten zu einer Renaissance der Phagentherapie [2]. Jedoch ist bis heute die Bakteriophagentherapie in Deutschland nur im Einzelfall als Heilversuch anwendbar. Aktuell gibt es kein zugelassenes Bakteriophagenprodukt zur klinischen Anwendung in der EU.

Präklinische Studien weisen darauf hin, dass die Phagentherapie das Potenzial besitzt, die Behandlung von knochen- bzw. implantatassoziierten Infektionen zu verbessern und zeigen Vorteile von Phagen gegenüber Antibiotika, wie Wirtsspezifität und geringe Toxizität für den Menschen auf [13]. Daher soll in diesem systematischen Review eine Übersicht bisheriger 
Tab. 1 Klinische Studien zur Anwendung von Bakteriophagen bei implantatassoziierten Infektionen

\begin{tabular}{|c|c|c|c|c|c|c|c|}
\hline $\begin{array}{l}\text { Autoren, } \\
\text { Jahr, Ort }\end{array}$ & $\begin{array}{l}\text { Indikation, } \\
\text { Stichproben- } \\
\text { größe, Alter und } \\
\text { Geschlecht }\end{array}$ & $\begin{array}{l}\text { Patho- } \\
\text { gene }\end{array}$ & $\begin{array}{l}\text { Phagentyp und Sen- } \\
\text { sitivität }\end{array}$ & Intervention & $\begin{array}{l}\text { Phagenkonzen- } \\
\text { tration }\end{array}$ & Ergebnisse & Nebenwirkungen \\
\hline $\begin{array}{l}\text { Cano } \\
\text { et al. } \\
2020 \text {, } \\
\text { USA, [3] }\end{array}$ & $\begin{array}{l}\text { PJI, } n=1 ; 62 \text { Jah- } \\
\text { re, } m\end{array}$ & $\begin{array}{l}\text { Klebsiella } \\
\text { pneu- } \\
\text { moniae }\end{array}$ & $\begin{array}{l}\text { KpJH46ழ (Adaptive } \\
\text { Phage Therapeutics } \\
\text { [APT], Gaithersburg, } \\
\text { MD, USA) }\end{array}$ & $\begin{array}{l}\text { Intravenöse } \\
\text { Anwendung, } \\
\text { zusätzlich } \\
\text { Antibiotikathe- } \\
\text { rapie }\end{array}$ & $\begin{array}{l}40 \text { tägliche In- } \\
\text { fusionen } 50 \mathrm{ml} \\
\left(6 \times 10^{10} \mathrm{PFU}\right) \text { in } \\
0,9 \% \text { Kochsalzlö- } \\
\text { sung }\end{array}$ & $\begin{array}{l}\text { Infekteradikation } \\
\text { Follow Up: } \\
\text { 8,5 Monate }\end{array}$ & Keine berichtet \\
\hline $\begin{array}{l}\text { Doub } \\
\text { et al. } \\
2020 \text {, } \\
\text { USA, [7] }\end{array}$ & $\begin{array}{l}\text { PJl, } n=1 ; 72 \text { Jah- } \\
\text { re, } m\end{array}$ & $\begin{array}{l}\text { S. au- } \\
\text { reus } \\
\text { (MRSA) }\end{array}$ & $\begin{array}{l}\text { Anti-Staphylococ- } \\
\text { ci-Phage SaGR51 } \varphi \\
\text { (Adaptive Phage The- } \\
\text { rapeutics), Sensitivität } \\
\text { extern bestätigt }\end{array}$ & $\begin{array}{l}\text { Intraartikuläre } \\
\text { Anwendung } \\
\text { und tägliche } \\
\text { intravenöse } \\
\text { Gabe, zusätz- } \\
\text { lich Antibio- } \\
\text { tikatherapie }\end{array}$ & $\begin{array}{l}\text { Intraartikulär } \\
\text { zwei Dosen } \\
5,4 \times 10^{9} \text { PFU } \\
\text { in } 10 \mathrm{ml} \mathrm{Kochsalz-} \\
\text { lösung; intravenös } \\
2,7 \times 10^{9} \text { PFU in } \\
50 \mathrm{ml} \mathrm{Kochsalzlö-} \\
\text { sung }\end{array}$ & $\begin{array}{l}\text { Infekteradikation } \\
\text { Follow Up: } 8 \text { Mo- } \\
\text { nate }\end{array}$ & $\begin{array}{l}\text { Nach dritter in- } \\
\text { travenöser Dosis } \\
\text { erhöhte Werte } \\
\text { von Aspartat- } \\
\text { Aminotransfera- } \\
\text { se, Alanin-Ami- } \\
\text { notransferase und } \\
\text { Abbruch der intra- } \\
\text { venösen Bakterio- } \\
\text { phagentherapie }\end{array}$ \\
\hline $\begin{array}{l}\text { Ferry } \\
\text { et al. } \\
2020, \\
\text { Frank- } \\
\text { reich, } \\
{[11]}\end{array}$ & $\begin{array}{l}\text { PJI, } n=1 ; 49 \text { Jah- } \\
\text { re, } \mathrm{m}\end{array}$ & $\begin{array}{l}\text { S. au- } \\
\text { reus } \\
\text { (MSSA) }\end{array}$ & $\begin{array}{l}\text { Zwei Anti-Staphylo- } \\
\text { cocci-Phagen (PP1493 } \\
\text { und PP1815), (Phere- } \\
\text { cydes Pharma, Nantes, } \\
\text { Frankreich), Sensitivi- } \\
\text { tät bestätigt }\end{array}$ & $\begin{array}{l}\text { Lokale An- } \\
\text { wendung } \\
\text { von } D A C^{\circledR} \\
\text { Hydrogel als } \\
\text { Phagenträ- } \\
\text { ger, zusätzlich } \\
\text { Antibiotikathe- } \\
\text { rapie }\end{array}$ & $\begin{array}{l}300 \mathrm{mg} \text { steriles } \\
\mathrm{DAC}{ }^{\circledR} \text {-Pulver ge- } \\
\text { füllt mit } 1 \mathrm{ml} \text { jeder } \\
\text { Bakteriophage } \\
\left(10^{10} \mathrm{PFU} / \mathrm{ml}\right) \\
\text { und } 5 \mathrm{ml} \text { sterilem } \\
\text { Wasser }\end{array}$ & Amputation & Keine berichtet \\
\hline $\begin{array}{l}\text { Ferry } \\
\text { et al. } \\
2020, \\
\text { Frank- } \\
\text { reich, } \\
\text { [10] }\end{array}$ & $\begin{array}{l}\text { PJI, } n=3 ; 80 \text { Jah- } \\
\text { re, m; } 84 \text { Jahre, } \\
\text { m; } 83 \text { Jahre, w }\end{array}$ & $\begin{array}{l}\text { S. au- } \\
\text { reus } \\
\text { (MSSA) }\end{array}$ & $\begin{array}{l}\text { Drei Anti-Staphylococ- } \\
\text { ci-Phagen (PP1493, } \\
\text { PP1815, PP1957), } \\
\text { (Pherecydes Pharma), } \\
\text { Sensitivität bestätigt }\end{array}$ & $\begin{array}{l}\text { Intraoperative } \\
\text { Injektion ins } \\
\text { Gelenk, zusätz- } \\
\text { liche Antibio- } \\
\text { tikatherapie }\end{array}$ & $\begin{array}{l}\text { Einmalig } \\
1 \times 10^{9} \mathrm{PFU} / \mathrm{ml}\end{array}$ & $\begin{array}{l}\text { Infekteradikation } \\
\text { in zwei Patien- } \\
\text { ten. Follow-Up: } \\
7 \text { Monate und } \\
2,5 \text { Jahre. Beste- } \\
\text { hende Fistel bei } \\
n=1 \text {. Follow Up: } \\
11 \text { Monate }\end{array}$ & Keine berichtet \\
\hline $\begin{array}{l}\text { Tkhilaish- } \\
\text { vili et al. } \\
2020, \\
\text { Deutsch- } \\
\text { land, [27] }\end{array}$ & $\begin{array}{l}\text { PJl, } n=1 ; 80 \text { Jah- } \\
\text { re, w }\end{array}$ & $\begin{array}{l}\text { Pseudo- } \\
\text { monas } \\
\text { aeru- } \\
\text { ginosa }\end{array}$ & $\begin{array}{l}\text { Anti-Pseudomonas- } \\
\text { Phage, (Eliava Insti- } \\
\text { tute, Tiflis, Georgien), } \\
\text { Sensitivität bestätigt }\end{array}$ & $\begin{array}{l}\text { Lokale Anwen- } \\
\text { dung intraope- } \\
\text { rativ und wie- } \\
\text { derholt über } \\
\text { Drainagen, } \\
\text { zusätzliche } \\
\text { Antibiotikathe- } \\
\text { rapie }\end{array}$ & $\begin{array}{l}\text { Lokal } 100 \mathrm{ml} \\
\left(10^{9} \mathrm{PFU} / \mathrm{ml}\right), \\
\text { dann } 5 \mathrm{ml}\left(10^{8}\right. \\
\text { PFU/ml) alle } 8 \mathrm{~h} \\
\text { über } 5 \text { Tage }\end{array}$ & $\begin{array}{l}\text { Infekteradika- } \\
\text { tion. Follow Up: } \\
10 \text { Monate }\end{array}$ & Keine berichtet \\
\hline \multirow[t]{2}{*}{$\begin{array}{l}\text { Onsea } \\
\text { et al. } \\
2019, \\
\text { Belgien, } \\
{[21]}\end{array}$} & \multirow[t]{2}{*}{$\begin{array}{l}\text { FRI, } n=4 \text {; Alter } \\
\text { und Geschlecht } \\
\text { nicht berichtet }\end{array}$} & \multirow{2}{*}{ 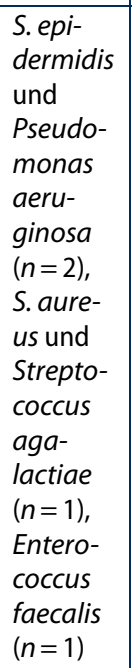 } & \multirow{2}{*}{$\begin{array}{l}\text { BFC1-Cocktail (Queen } \\
\text { Astrid Military Hospi- } \\
\text { tal, Brüssel, Belgien) } \\
\text { und „Pyo-Phage“- } \\
\text { Cocktail (Eliava Ins- } \\
\text { titute), Sensitivität } \\
\text { bestätigt }\end{array}$} & \multirow{2}{*}{$\begin{array}{l}\text { Lokale, in- } \\
\text { traoperative } \\
\text { Spülung, Im- } \\
\text { plantation } \\
\text { eines Genta- } \\
\text { micin-imprä- } \\
\text { gnierter Kolla- } \\
\text { genschwamm } \\
\text { getränkt in } \\
\text { Phagenlösung, } \\
\text { postoperativ } \\
\text { wiederholte } \\
\text { Anwendung } \\
\text { über Draina- } \\
\text { ge, zusätzlich } \\
\text { Antibiotikathe- } \\
\text { rapie }\end{array}$} & $\begin{array}{l}\text { Lokal } 10-40 \mathrm{ml} \\
\left(10^{7} \mathrm{PFU} / \mathrm{ml} \text { in }\right. \\
0,9 \% \text { Kochsalzlö- } \\
\text { sung) }\end{array}$ & \multirow[t]{2}{*}{$\begin{array}{l}\text { Infekteradikation } \\
\text { in allen Fäl- } \\
\text { len. Follow Up: } \\
\text { 8-16 Monate }\end{array}$} & \multirow{2}{*}{$\begin{array}{l}\text { Ein Patient zeigte } \\
\text { lokale Rötung und } \\
\text { Schmerzen wäh- } \\
\text { rend der Adminis- } \\
\text { tration des „Pyo- } \\
\text { Phage“-Cocktails } \\
\text { über die Drainage }\end{array}$} \\
\hline & & & & & $\begin{array}{l}\text { Postoperativ } \\
\text { 3-mal täglich für } \\
\text { 7-10 Tage, mit } \\
\text { individuellen } \\
\text { Protokollen }\end{array}$ & & \\
\hline
\end{tabular}




\begin{tabular}{|c|c|c|c|c|c|c|c|}
\hline $\begin{array}{l}\text { Autoren, } \\
\text { Jahr, Ort }\end{array}$ & $\begin{array}{l}\text { Indikation, } \\
\text { Stichproben- } \\
\text { größe, Alter und } \\
\text { Geschlecht }\end{array}$ & $\begin{array}{l}\text { Patho- } \\
\text { gene }\end{array}$ & $\begin{array}{l}\text { Phagentyp und Sen- } \\
\text { sitivität }\end{array}$ & Intervention & $\begin{array}{l}\text { Phagenkonzen- } \\
\text { tration }\end{array}$ & Ergebnisse & Nebenwirkungen \\
\hline $\begin{array}{l}\text { Nir-Paz } \\
\text { et al. } \\
2019 \text {, } \\
\text { Israel, } \\
{[20]}\end{array}$ & $\begin{array}{l}\text { FRI, } n=1 ; \\
\text { 42 Jahre, } m\end{array}$ & $\begin{array}{l}\text { Acineto- } \\
\text { bacter } \\
\text { bau- } \\
\text { mannii, } \\
\text { Kleb- } \\
\text { siella } \\
\text { pneu- } \\
\text { moniae }\end{array}$ & $\begin{array}{l}\text { Phagen AbKT21 } 43 \\
\text { und KpKT21 } 11 \text { (US- } \\
\text { Naval Medical Re- } \\
\text { search Centre Phage } \\
\text { Bank), Sensitivität } \\
\text { bestätigt }\end{array}$ & $\begin{array}{l}\text { Wiederholte } \\
\text { intravenöse } \\
\text { Verabreichung, } \\
\text { zusätzlich } \\
\text { Antibiotikathe- } \\
\text { rapie }\end{array}$ & $\begin{array}{l}1 \text { ml jeder Pha- } \\
\text { ge (Titer nicht } \\
\text { berichtet) für } \\
35 \text { min über } 5 \text { Ta- } \\
\text { ge. Nach einer } \\
\text { Woche nochmals } \\
1 \text { ml der Anti-A.- } \\
\text { baumannii-Phage } \\
\text { über } 6 \text { Tage }\end{array}$ & $\begin{array}{l}\text { Infekteradikation. } \\
\text { Follow Up: } 8 \text { Mo- } \\
\text { nate }\end{array}$ & Keine berichtet \\
\hline $\begin{array}{l}\text { Ferry } \\
\text { et al. } \\
2018, \\
\text { Frank- } \\
\text { reich, [9] }\end{array}$ & $\begin{array}{l}\text { PJl, } n=1 ; 80 \text { Jah- } \\
\text { re, w }\end{array}$ & $\begin{array}{l}\text { Pseudo- } \\
\text { monas } \\
\text { aerugi- } \\
\text { nosa, } \\
\text { S. au- } \\
\text { reus }\end{array}$ & $\begin{array}{l}\text { Zwei individualisier- } \\
\text { te Phagencocktails } \\
\text { (jeweils drei Phagen) } \\
\text { (Pherecydes Pharma), } \\
\text { Sensitivität für Anti- } \\
\text { Pseudomonas-Phage } \\
\text { und } 2 / 3 \text { Anti-Sta- } \\
\text { phylococcus-Phagen } \\
\text { bestätigt }\end{array}$ & $\begin{array}{l}\text { Intraoperative } \\
\text { Injektion ins } \\
\text { Gelenk, zusätz- } \\
\text { liche Antibio- } \\
\text { tikatherapie }\end{array}$ & $\begin{array}{l}\text { Einmalig } 6 \mathrm{ml} \text { je- } \\
\text { des Phagen-Cock- } \\
\text { tails }\left(10^{10} \mathrm{PFU} / \mathrm{ml}\right) \\
\text { in jeweils } 10 \mathrm{ml} \\
\text { Kochsalzlösung }\end{array}$ & $\begin{array}{l}\text { Infekteradikation. } \\
\text { Follow Up } 18 \text { Mo- } \\
\text { nate }\end{array}$ & Keine berichtet \\
\hline $\begin{array}{l}\text { Ferry } \\
\text { et al. } \\
2018, \\
\text { Frank- } \\
\text { reich, [8] }\end{array}$ & $\begin{array}{l}\text { Infektion des } \\
\text { Iliosakralgelenks } \\
\text { nach Zement- } \\
\text { augmentation } \\
\text { in Folge einer } \\
\text { Chemotherapie } \\
\text { bei Lungenkar- } \\
\text { zinom, } n=1 ; \\
\text { Anfang } 60, \mathrm{~m}\end{array}$ & $\begin{array}{l}\text { Pseudo- } \\
\text { monas } \\
\text { aeru- } \\
\text { ginosa }\end{array}$ & $\begin{array}{l}\text { Individualisierter Pha- } \\
\text { gen-Cocktail (4 Pha- } \\
\text { gen) (Pherecydes } \\
\text { Pharma), Sensitivität } \\
\text { bestätigt }\end{array}$ & $\begin{array}{l}\text { Lokale, in- } \\
\text { traoperative } \\
\text { Anwendung, } \\
\text { lokale Wun- } \\
\text { danwendung, } \\
\text { zusätzliche } \\
\text { Antibiotika- } \\
\text { therapie (lokal } \\
\text { und intrave- } \\
\text { nös) }\end{array}$ & $\begin{array}{l}1,2-9,7 \times 10^{8} \\
\text { PFU/ml in } 30 \mathrm{ml} \\
\text { Kochsalzlösung }\end{array}$ & $\begin{array}{l}\text { Infekteradikation, } \\
\text { Patient verstarb } \\
\text { am Lungenkar- } \\
\text { zinom an Tag } 45 \\
\text { postoperativ }\end{array}$ & Keine berichtet \\
\hline \multirow[t]{3}{*}{$\begin{array}{l}\text { Patey } \\
\text { et al. } \\
2018, \\
\text { Frank- } \\
\text { reich, } \\
{[22]}\end{array}$} & $\begin{array}{l}\text { FRI, } n=4 ; \\
44 \text { Jahre, m; } \\
68 \text { Jahre, w; } \\
25 \text { Jahre, m; } \\
40 \text { Jahre, w }\end{array}$ & $\begin{array}{l}\text { S. au- } \\
\text { reus } \\
(n=6)\end{array}$ & \multirow[t]{3}{*}{$\begin{array}{l}\text { "Pyo-Phage"-Cocktail } \\
\text { (Eliava Institute und } \\
\text { Microgen, Moskau, } \\
\text { Russland) }\end{array}$} & \multirow{3}{*}{$\begin{array}{l}\text { Postoperative } \\
\text { Anwendung, } \\
\text { nicht weiter } \\
\text { spezifiziert, } \\
\text { zusätzliche } \\
\text { Antibiotikathe- } \\
\text { rapie }\end{array}$} & \multirow[t]{3}{*}{ Nicht berichtet } & \multirow[t]{2}{*}{$\begin{array}{l}\text { Infekteradikation } \\
\text { in } 6 / 8 \text { Fällen }\end{array}$} & \multirow[t]{3}{*}{ Keine berichtet } \\
\hline & $\begin{array}{l}\text { Osteomyelitis, } \\
n=1 ; 84 \text { Jahre, } \\
\mathrm{m}\end{array}$ & $\begin{array}{l}\text { S. aure- } \\
\text { us und } \\
\text { Pseudo- } \\
\text { monas } \\
\text { aeru- } \\
\text { ginosa } \\
(n=1)\end{array}$ & & & & & \\
\hline & $\begin{array}{l}\text { PJI, } n=3 ; 72 \text { Jah- } \\
\text { re, w; } 80 \text { Jahre, w }\end{array}$ & $\begin{array}{l}\text { Pseudo- } \\
\text { monas } \\
\text { aeru- } \\
\text { ginosa } \\
(n=1)\end{array}$ & & & & $\begin{array}{l}\text { Keimwechsel in } \\
\text { zwei Fällen }\end{array}$ & \\
\hline $\begin{array}{l}\text { Vogt } \\
\text { et al. } \\
2017, \\
\text { Deutsch- } \\
\text { land, [31] }\end{array}$ & $\begin{array}{l}\text { PJl, } n=1 ; 33 \text { Jah- } \\
\text { re, } m\end{array}$ & $\begin{array}{l}\text { Pseudo- } \\
\text { monas } \\
\text { aerugi- } \\
\text { nosa, } \\
\text { Acineto- } \\
\text { bacter } \\
\text { bau- } \\
\text { mannii }\end{array}$ & $\begin{array}{l}\text { "Pyo-Phage“-Cocktail } \\
\text { (Eliava Institute), } \\
\text { Sensitivität bestätigt }\end{array}$ & $\begin{array}{l}\text { Wiederholte } \\
\text { Injektion „Pyo- } \\
\text { Phage“-Cock- } \\
\text { tail über Drai- } \\
\text { nagen }\end{array}$ & Nicht berichtet & Amputation & Keine berichtet \\
\hline
\end{tabular}




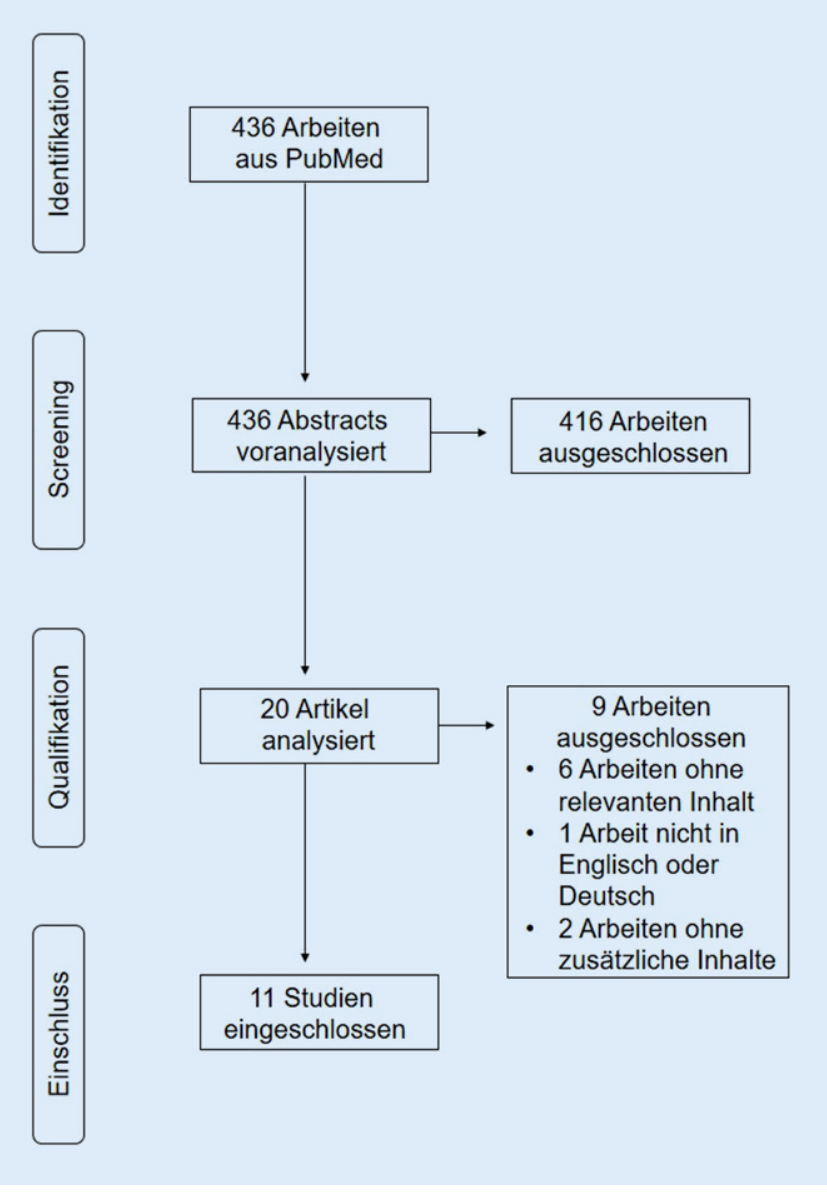

\section{Ergebnisse}

Die systematische Suche ergab 436 Treffer. Nach Anwendung der Ausschlusskriterien verblieben 20 Studien, die anhand des Volltextes überprüft wurden. Hiervon wurden 9 weitere ausgeschlossen, $n=6$ aufgrund nicht relevanter Daten für die Studie, $n=1$ aufgrund keiner Verfügbarkeit in englischer Sprache und $n=2$, die Daten beinhalteten, die bereits in einer eingeschlossenen Publikation berichtet wurden. Insgesamt wurden 11 Studien in die Übersichtsarbeit eingeschlossen (• Tab. 1; - Abb. 2; [3, 8-12, 21-23, 28, 32]).

Durchgeführt wurden die Studien in Frankreich $(n=5)$, den Vereinigten Staaten $(n=2)$, Deutschland $(n=2)$, Israel $(n=1)$ und Belgien $(n=1)$. Identifiziert wurden $n=8$ Fallberichte und $n=3$ Fallserien. Insgesamt wurden die Daten von 23 Patienten berichtet. Indikationen der Phagentherapie waren periprothetische Infektionen $(n=12,52,2 \%)$, frakturassoziierte Infektionen $(n=9,39,1 \%)$, Osteomyelitis $(n=1$, $4,4 \%$ ) und eine Iliosakralgelenkinfektion nach Zementaugmentation einer Metastase $(n=1,4,4 \%)$. Die zugrundeliegende Infektion war monobakteriell in 16 der Fälle $(69,6 \%)$ und polybakteriell in 7 Fällen $(30,4 \%)$. Die häufigsten Erreger waren Staphylococcus aureus ( $n=14,46,7 \%$ ) und Pseudomonas aeruginosa ( $n=8,26,7 \%$ ). Ein vorheriger Sensitivitätstest der Phagen hinsichtlich der zugrundeliegenden Keime wurde in 9/11 Artikeln berichtet. Die Phagen wurden intravenös verabreicht, intraoperativ ins Gelenk injiziert, intraoperativ lokal angewendet oder über Drainagen appliziert. Zehn von elf Studien $(90,9 \%)$ berichten eine ergänzende Antibiotikatherapie. Keine der Studien erlaubte eine Unterscheidung zwischen Effekten durch die Phagen und denen der Antibiotikatherapie. In neun Studien wurden keine Nebenwirkungen der Phagentherapie berichtet (Patienten: $n=21 ; 91,3 \%$ ). Ein Patient zeigte eine lokale Rötung und Schmerzen während der Administration des Phagencocktails über die Drainage [22]. Bei einem weiteren traten nach dritter intravenöser Dosis erhöhte Leberwerte (Aspartat-Aminotransferase und Alanin-Aminotransferase) auf, woraufhin die intravenöse Bakteriophagentherapie abgebrochen wurde [8]. Eine vollständige Infekteradikation über 
mindestens 7 Monate konnte bei $18 \mathrm{~Pa}$ tienten $(78,3 \%)$ erreicht werden. Bei zwei Fällen erfolgte ein Keimwechsel [23] und bei einem Patienten blieb eine Fistel bestehen [11]. In zwei Fallstudien erfolgte kein Extremitätenerhalt durch die Phagentherapie $[12,32]$.

\section{Diskussion}

Diese Arbeit gibt eine systematische Übersicht über den klinischen Einsatz der Phagentherapie bei Knochen- und Protheseninfektionen. Insgesamt wurden seit dem Jahr 200023 Fälle mit einer Knochenoder Protheseninfektion berichtet, die mit Bakteriophagen therapiert wurden. Bei $78,3 \%$ der 22 Patienten konnte eine vollständige Infekteradikation für mindestens 7 Monate erreicht werden. In 91,3\% der Fälle konnten keine Nebenwirkungen der Bakteriophagentherapie beobachtet werden.

In Anbetracht der steigenden Antibiotikamultiresistenz erscheint der Ansatz, sich die natürlichen Feinde der Bakterien - nämlich die Bakteriophagen therapeutisch zu Nutze zu machen, naheliegend und erfolgsversprechend [19]. Eine beträchtliche Anzahl von 11,6- 22,6\% der periprothetischen Gelenkinfektionen werden durch sogenannte „Difficult-totreat"(DTT)-Pathogene verursacht, die resistent gegenüber biofilmaktiven Antibiotika sind [31, 37]. DTT-Infektionen sind mit einer erhöhten Mortalität verbunden, führen zu schlechteren klinischen Ergebnissen und sind mit höheren Revisionsraten vergesellschaftet [31,35]. Auch polymikrobielle Infektionen verkomplizieren den Behandlungsverlauf periprothetischer Infektionen [34]. Hier erscheint es zielführend, Phagen in Kombination mit Antibiotikatherapie anzuwenden.

Neben dem therapeutischen Nutzen, welcher durch eine additive Bakteriophagentherapie in präklinischen Studien impliziert wird, könnten Phagen auch zur Prävention implantatassoziierter Infektionen eingesetzt werden [24]. Hier bestehen diverse Möglichkeiten der Beladung von Materialien wie Keramik, K-Drähte [17] oder Hydrogel [18]. Jedoch waren die identifizierten Studien gering in ihrer Anzahl und zeigten eine Inhomogenität sowohl bezüglich (1) der Indikation, (2) der Pa- thogene und (3) der Art der Anwendung. Letztere erfolgte intravenös, intraartikulär, lokal oder über Drainagen mit unterschiedlicher Dauer. Dabei war die Dosis, der Zeitpunkt und die Häufigkeit der Verabreichung heterogen in der Literatur. Da Bakteriophagen in der EU nicht zur klinischen Anwendung zugelassen sind, handelt es sich bei den 11 identifizierten Studien um individuelle Heilversuche, aus welchen die optimale Behandlung mit Phagen nicht ableitbar ist. Zusätzlich unterschied keine der Studien zwischen Effekten durch die Phagen und denen der Antibiotikatherapie.

Für einen nächsten Schritt in Richtung effektiver Anwendung der Phagentherapie ist somit die Etablierung von vereinheitlichten Behandlungsprotokollen und internationalen Richtlinien unerlässlich [7]. Bei der systematischen Suche konnte keine randomisierte kontrollierte Studie identifiziert werden, welche die genannten Punkte adressiert. Derzeit ist lediglich eine klinische Studie zur Evaluierung der Sicherheit der Phagentherapie bei Knochen- und Protheseninfektionen registriert (https://clinicaltrials.gov/, Juni 2021). Um die Möglichkeiten der Bakteriophagentherapie zu evaluieren werden zukünftige klinische Studien mit höherem Evidenzgrad benötigt. Zusätzlich ist die routinemäßige und zeitnahe Gewinnung von Phagen und die Herstellung der Phagencocktails unter "Good Manufacturing Practice"-Bedingungen als Herausforderung zu betrachten. Zudem wird ein großer Teil der Erkenntnisse zur Phagentherapie von einer sehr begrenzten Anzahl von Phagenisolaten abgeleitet, was die weltweite Akzeptanz von Bakteriophagen als Therapeutika verlangsamt. Von den $10^{31}$ existierenden Phagen, wurden bisher weniger als $10^{4}$ isoliert und sequenziert (https://www.ncbi.nlm.nih.gov/, Juni 2021), weshalb die Weiterentwicklung von kulturunabhängigen Methoden in den Fokus gerückt werden sollte [16].

\section{Fazit für die Praxis}

- In Anbetracht der steigenden Antibiotikamultiresistenz werden alternative Therapieansätze benötigt.

- Bakteriophagen sind eine vielversprechende Behandlungsmethode von Kno- chen- und Gelenksinfektionen in Kombination mit Antibiotikatherapie.

- Die Etablierung von optimalen Behandlungsprotokollen ist erforderlich.

- Zukünftige klinische Studien mit höherem Evidenzgrad werden für eine erfolgreiche Translation der Bakteriophagentherapie in die klinische Praxis benötigt.

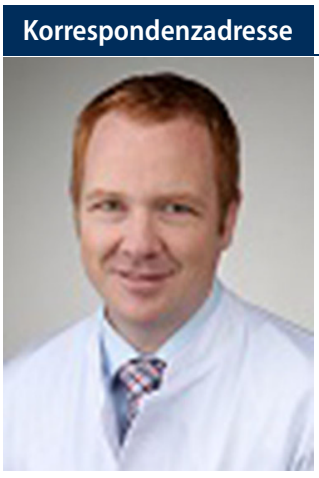

PD Dr. med. Markus Rupp

Klinik und Poliklinik für Unfallchirurgie, Universitätsklinikum Regensburg

Franz-Josef-Strauß-Allee 11, 93053 Regens-

burg, Deutschland

markus.rupp@ukr.de

Funding. Open Access funding enabled and organized by Projekt DEAL.

\section{Einhaltung ethischer Richtlinien}

Interessenkonflikt. N. Walter, L. Deng, C. Brochhausen, V. Alt und M. Rupp geben an, dass kein Interessenkonflikt besteht.

Für diesen Beitrag wurden von den Autoren keine Studien an Menschen oder Tieren durchgeführt. Für die aufgeführten Studien gelten die jeweils dort angegebenen ethischen Richtlinien.

Open Access. Dieser Artikel wird unter der Creative Commons Namensnennung 4.0 International Lizenz veröffentlicht, welche die Nutzung, Vervielfältigung, Bearbeitung, Verbreitung und Wiedergabe in jeglichem Medium und Format erlaubt, sofern Sie den/die ursprünglichen Autor(en) und die Quelle ordnungsgemäß nennen, einen Link zur Creative Commons Lizenz beifügen und angeben, ob Änderungen vorgenommen wurden.

Die in diesem Artikel enthaltenen Bilder und sonstiges Drittmaterial unterliegen ebenfalls der genannten Creative Commons Lizenz, sofern sich aus der Abbildungslegende nichts anderes ergibt. Sofern das betreffende Material nicht unter der genannten Creative Commons Lizenz steht und die betreffende Handlung nicht nach gesetzlichen Vorschriften erlaubt ist, ist für die oben aufgeführten Weiterverwendungen des Materials die Einwilligung des jeweiligen Rechteinhabers einzuholen. 
Weitere Details zur Lizenz entnehmen Sie bitte der Lizenzinformation auf http://creativecommons.org/ licenses/by/4.0/deed.de.

\section{Literatur}

1. Akanda ZZ, Taha M, Abdelbary H (2018) Current review. The rise of bacteriophage as a unique therapeutic platform in treating peri-prosthetic joint infections. J Orthop Res 36(4):1051-1060. https://doi.org/10.1002/jor.23755

2. Aslam B, Wang W, Arshad Ml, Khurshid M, Muzammil S, Rasool MH, Nisar MA, Alvi RF, Aslam MA, Qamar MU, Salamat MKF, Baloch Z (2018) Antibiotic resistance: a rundown of a global crisis. Infect Drug Resist 11:1645-1658. https://doi. org/10.2147/IDR.S173867

3. Cano EJ, Caflisch KM, Bollyky PL, van Belleghem JD, Patel R, Fackler J, Brownstein MJ, Horne B, Biswas B, Henry M, Malagon F, Lewallen DG, Suh GA (2020) Phage therapy for limb-threatening prosthetic knee Klebsiella pneumoniae infection: case report and in vitro characterization of anti-biofilm activity. Clin Infect Dis. https://doi.org/10.1093/ $\mathrm{cid} / \mathrm{ciaa} 705$

4. Chanishvili N (2012) Phage therapy-history from Twort and d'Herelle through Soviet experience to currentapproaches. Adv Virus Res 83:3-40. https:// doi.org/10.1016/B978-0-12-394438-2.00001-3

5. Clokie MR, Millard AD, Letarov AV, Heaphy S (2011) Phages in nature. Bacteriophage 1(1):31-45. https://doi.org/10.4161/bact.1.1.14942

6. D'Herelle $F$ (2007) On an invisible microbe antagonistic toward dysenteric bacilli: brief note by Mr. F. D'Herelle, presented by Mr. Roux. 1917. Res Microbiol 158(7):553-554. https://doi.org/10. 1016/j.resmic.2007.07.005

7. Doub JB (2020) Bacteriophage therapy for clinical biofilm infections: parameters that influence treatment protocols and current treatment approaches. Antibiotics (Basel) 9(11):799. https:// doi.org/10.3390/antibiotics9110799

8. Doub JB, Ng VY, Johnson AJ, Slomka M, Fackler J, Horne B, Brownstein MJ, Henry M, Malagon F, Biswas B (2020) Salvage bacteriophage therapy for a chronic MRSA prosthetic joint infection. Antibiotics (Basel) 9(5):241. https://doi.org/10. 3390/antibiotics9050241

9. Ferry T, Boucher F, Fevre C, Perpoint $T$, Chateau J, Petitjean C, Josse J, Chidiac C, L'hostis G, Leboucher G, Laurent F (2018) Innovations for the treatment of a complex bone and joint infection due to XDR Pseudomonas aeruginosa including local application of a selected cocktail of bacteriophages. J Antimicrob Chemother 73(10):2901-2903. https://doi.org/10.1093/jac/ dky263

10. Ferry T, Leboucher G, Fevre C, Herry Y, Conrad A, Josse J, Batailler C, Chidiac C, Medina M, Lustig S, Laurent $F$ (2018) Salvage debridement, antibiotics and implant retention ("DAIR") with local injection of a selected cocktail of bacteriophages: Is it an option for an elderly patient with relapsing staphylococcus aureus prosthetic-joint infection? Open Forum Infect Dis 5(11):ofy269. https://doi. org/10.1093/ofid/ofy269

11. Ferry T, Kolenda C, Batailler C, Gustave C-A, Lustig S, Malatray M, Fevre C, Josse J, Petitjean C, Chidiac C, Leboucher G, Laurent F (2020) Phage therapy as adjuvant to conservative surgery and antibiotics to salvage patients with relapsing $S$. aureus prosthetic

Treatment of bone and periprosthetic infections with bacteriophages. A systematic review

Background: The treatment of bone and prosthesis infections remains difficult despite modern treatment concepts. Further tools for the improvement of treatment outcome are desirable. Preclinical studies provide promising evidence of the efficacy of bacteriophages for the treatment of bone and joint infections.

Objectives: The present work provides a systematic review of the clinical application of bacteriophages for the treatment of bone and joint infections.

Materials and methods: A systematic search was performed in PubMed to identify primary clinical data on the use of phage therapy in patients with bone and joint infection.

Results: Eight case reports and three case series were included in the study. Indications for phage therapy were periprosthetic joint infection $(n=12,52.2 \%)$, fracture-related infection ( $n=9,39.1 \%)$, osteomyelitis $(n=1,4.4 \%)$ and sacroiliac joint infection after cement augmentation of a metastasis $(n=1,4.4 \%)$. Interventions were heterogeneous; phages were administered intravenously, injected intraoperatively into the joint, applied locally intraoperatively, or administered via drains. In combination with antibiotic therapy, complete infection eradication was achieved in 18 patients (78.3\%). No side effects were reported in $91.3 \%$ of patients.

Conclusion: Bacteriophages represent a promising treatment option for bone and prosthesis infections in combination with antibiotic therapy. Future clinical trials with a higher level of evidence are required for the successful translation of bacteriophage therapy into clinical practice.

\section{Keywords}

Alternative therapy - Antibiotic resistance - Phage therapy - Periprosthetic infection - Treatment outcome

knee infection. Front Med (Lausanne) 7:570572. https://doi.org/10.3389/fmed.2020.570572

12. Ferry T, Batailler C, Petitjean C, Chateau J, Fevre C, Forestier $\mathrm{E}$, Brosset $\mathrm{S}$, Leboucher $\mathrm{G}$, Kolenda $\mathrm{C}$, Laurent F, Lustig S (2020) The potential innovative use of bacteriophages within the DAC ${ }^{\circledR}$ hydrogel to treat patients with knee megaprosthesis infection requiring "debridement antibiotics and implant retention" and soft tissue coverage as salvage therapy. Front Med (Lausanne) 7:342. https://doi. org/10.3389/fmed.2020.00342

13. Gibb BP, Hadjiargyrou M (2021) Bacteriophage therapy for bone and joint infections. Bone Joint $J$ 103-B(2):234-244. https://doi.org/10.1302/0301 620X.103B2.BJJ-2020-0452.R2

14. Gristina AG (1987) Biomaterial-centered infection: microbial adhesion versus tissue integration. Science 237(4822):1588-1595. https://doi.org/10. $1126 /$ science. 3629258

15. Gristina AG, Costerton JW (1985) Bacterial adherence to biomaterials and tissue. The significance of its role in clinical sepsis. J Bone Joint Surg Am 67(2):264-273

16. Hayes S, Mahony J, Nauta A, van Sinderen D (2017) Metagenomic approaches to assess bacteriophages in various environmental niches. Viruses 9(6):127.https://doi.org/10.3390/v9060127

17. Kaur S, Harjai K, Chhibber S (2014) Bacteriophage mediated killing of Staphylococcus aureus in vitro on orthopaedic $\mathrm{K}$ wires in presence of linezolid prevents implant colonization. PLoS One 9(3):e90411. https://doi.org/10.1371/journal. pone.0090411

18. Kim HY, Chang RYK, Morales S, Chan H-K (2021) Bacteriophage-delivering hydrogels: current progressincombating antibiotic resistantbacterial infection. Antibiotics (Basel) 10(2):130. https://doi. org/10.3390/antibiotics10020130

19. Moelling K, Broecker F, Willy C (2018) A wakeup call: we need phage therapy now. Viruses 10(12):688. https://doi.org/10.3390/v10120688

20. Moher D, Liberati A, Tetzlaff J, Altman DG (2009) Preferred reporting items for systematic reviews and meta-analyses: the PRISMA statement. PLoS Med 6(7):e1000097. https://doi.org/10.1371/ journal.pmed.1000097

21. Nir-Paz R, Gelman D, Khouri A, Sisson BM, Fackler J, Alkalay-Oren S, Khalifa L, Rimon A, Yerushalmy O, Bader R, Amit S, Coppenhagen-Glazer S, Henry M, Quinones J, Malagon F, Biswas B, Moses AE, Merril G, Schooley RT, Brownstein MJ, Weil YA, Hazan R (2019) Successful treatment of antibioticresistant, poly-microbial bone infection with bacteriophages and antibiotics combination. Clin Infect Dis 69(11):2015-2018. https://doi.org/10. 1093/cid/ciz222

22. Onsea J, Soentjens $P$, Djebara $S$, Merabishvili $M$, Depypere $M$, Spriet I, de Munter $P$, Debaveye $Y$, Nijs S, Vanderschot $P$, Wagemans J, Pirnay J-P, Lavigne R, Metsemakers W-J (2019) Bacteriophage application for difficult-to-treat musculoskeletal infections: development of a standardized multidisciplinary treatment protocol. Viruses 11(10):891.https://doi.org/10.3390/v11100891

23. Patey O, McCallin S, Mazure H, Liddle M, Smithyman A, Dublanchet A (2018) Clinical indications and compassionate use of phage therapy:personal experience and literature review with a focus on osteoarticular infections. Viruses 11(1):18. https:// doi.org/10.3390/v11010018

24. Rotman SG, Sumrall E, Ziadlou R, Grijpma DW, Richards RG, Eglin D, Moriarty TF (2020) Local bac- 
teriophage delivery for treatment and prevention of bacterial infections. Front Microbiol 11:538060. https://doi.org/10.3389/fmicb.2020.538060

25. Slopek S, Weber-Dabrowska B, Dabrowski M, Kucharewicz-Krukowska A (1987) Results of bacteriophage treatment of suppurative bacterial infections in the years 1981-1986. Arch Immunol Ther Exp (Warsz) 35(5):569-583

26. Stewart PS, Costerton JW (2001) Antibiotic resistance of bacteria in biofilms. Lancet 358(9276):135-138. https://doi.org/10.1016/ s0140-6736(01)05321-1

27. Sulakvelidze A, Alavidze Z, Morris JG (2001) Bacteriophage therapy. Antimicrob Agents Chemother 45(3):649-659. https://doi.org/10.1128/AAC.45.3. 649-659.2001

28. Tkhilaishvili T, Winkler T, Müller M, Perka $C$, Trampuz A (2019) Bacteriophages as adjuvant to antibiotics for the treatment of periprosthetic joint infection caused by multidrug-resistant pseudomonas aeruginosa. Antimicrob Agents Chemother 64(1):e00924-19. https://doi.org/10. 1128/AAC.00924-19

29. TrampuzA, Widmer AF (2006) Infections associated with orthopedic implants. Curr Opin Infect Dis 19(4):349-356. https://doi.org/10.1097/01.qco. 0000235161.85925.e8

30. Trampuz A, Zimmerli W (2005) Prosthetic joint infections: update in diagnosis and treatment. Swiss Med Wkly 135(17-18):243-251

31. UII C, Yilmaz E, Baecker $\mathrm{H}$, Schildhauer TA, Waydhas C, Hamsen U (2020) Microbial findings and the role of difficult-to-treat pathogens in patients with periprosthetic infection admitted to the intensive care unit. Orthop Rev (Pavia) 12(3):8867. https://doi.org/10.4081/or.2020.8867

32. Vogt $D$, Sperling $S$, Tkhilaishvili T, Trampuz A, Pirnay J-P, Willy C (2017) „Beyond antibiotic therapy" - Zukünftige antiinfektiöse Strategien - Update 2017. Unfallchirurg 120(7):573-584. https://doi.org/10.1007/s00113-017-0374-6

33. Weber-Dabrowska B, Mulczyk M, Górski A (2000) Bacteriophage therapy of bacterial infections: an update of our institute's experience. Arch Immunol Ther Exp (Warsz) 48(6):547-55

34. Wimmer MD, Friedrich MJ, Randau TM, Ploeger MM, Schmolders J, Strauss AA, Hischebeth GTR, Pennekamp PH, Vavken P, Gravius S (2016) Polymicrobial infections reduce the cure rate in prosthetic joint infections: outcome analysis with two-stage exchange and follow-up $\geq$ two years. Int Orthop 40(7):1367-1373. https://doi.org/10 1007/s00264-015-2871-y

35. Wimmer MD, Hischebeth GTR, Randau TM, Gathen $M$, Schildberg FA, Fröschen FS, Kohlhof $H_{1}$ Gravius S (2020) Difficult-to-treat pathogens significantly reduce infection resolution in periprosthetic joint infections. Diagn Microbiol Infect Dis 98(2):115114. https://doi.org/10.1016/j. diagmicrobio.2020.115114

36. Zimmerli W, Trampuz A, Ochsner PE (2004) Prosthetic-joint infections. N Engl J Med 351(16):1645-1654. https://doi.org/10.1056/ NEJMra040181

37. Rupp M, Baertl S, Walter N, Hitzenbichler F, Ehrenschwender M, Alt V (2021) Is There a Difference in Microbiological Epidemiology and Effective Empiric Antimicrobial Therapy Comparing Frac ture-Related Infection and Periprosthetic Joint Infection? A Retrospective Comparative Study. Antibiotics 10(8):921

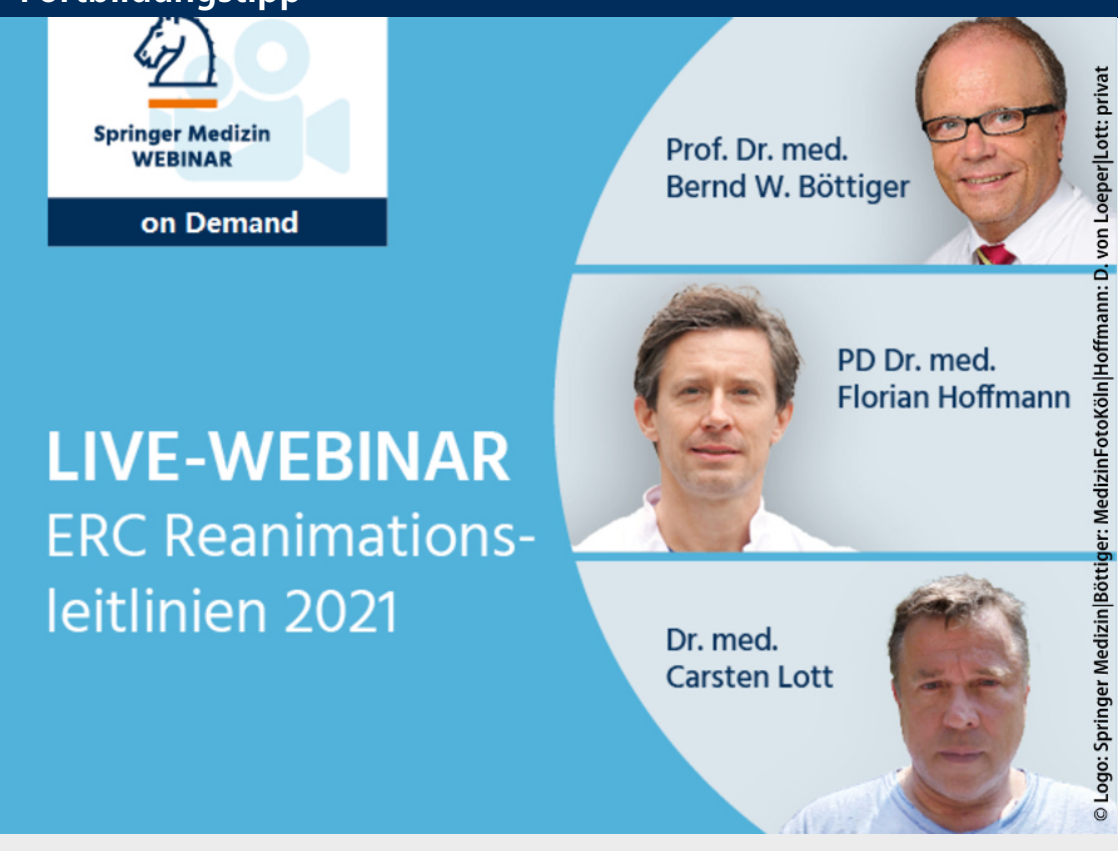

Fragen zur Reanimation von Kindern?

Ein Kind zu reanimieren ist auch für langjährige Ärtz*innen und Kolleg*innen des Rettungsdienstfachpersonals eine Herausforderungen. Oft stellen sich konkrete Fragen, die einen verunsichern. Lesen Sie hier kurz und knapp die Antworten von PD Dr. Florian Hoffmann auf einige Fragen Ihrer Kolleg*innen und die dazugehörigen Empfehlungen aus der ERC-Reanimationslinein 2021.

Das komplette Webinar zu den ERCReanimationsleitlinen

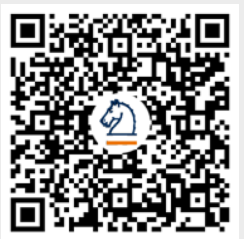

Im Webinar finden Sie die essentiellen Grundlagen der ERC-Reanimationsleitlinien zusammengefasst. Der Fokus liegt dabei auf Koronarthrombosen und Herzinfarkt und der der Renimation bei Kindern und Jugendlichen.

\section{Die Referenten:}

- Prof. Dr. B. W. Böttiger, Köln

- PD Dr. Florian Hoffman, München

- Dr. med Carsten Lott, Mainz

[URL: www.springermedizin.de/webinar-ercleitlinien]
Antworten aus 3 Fragen zum Webinar:

Erkennt der AED anhand des Körperwiderstands eine mögliche Reduktion der Energiezahl oder werden bei einem Säugling auch $\mathbf{3 6 0 \mathrm { J }}$ abgegeben?

> Bisher erkennt der AED nur, ob K-Elektroden oder E-Elektroden angeschlossen sind. In Zukunft wird aber eine Berechnung nach Körperwiderstand realisiert werden können.

Gibt es eine Empfehlungen zum Zeitpunkt der Intubation unter CPR? > Die Intubation ohne Effekt auf Outcome ist sehr in den Hintergrund gerückt und nur dann durchführbar, wenn alle notwendigen Maßnahmen laufen und ein Experte für Kinderintubation anwesend ist ( $>100-150$ Intubationen).

Gibt es eine Evidenz zur Verwendung von Erwachsenen-Masken bei Kindern, im Sinne einer Full-Face-Alternative, falls kein Kinderbeutel verfügbar ist? >> Es gibt keine Evidenz aber Erfahrungen mit Drehen der Maske um $180 \mathrm{Grad}$, mit Einmalmasken mit aufblasbarem Rand kann auch mit Erwachsenen-Masken zumeist eine gute Ventilation erreicht werden. 